\title{
SAINT VENANT'S PRINCIPLE IN ORTHOTROPIC PLANAR ELASTICITY: RATES-OF-DIFFUSION FOR STRESS
}

BY

\author{
W. J. STRONGE AND M. KASHTALYAN
}

\section{Department of Engineering, University of Cambridge, Cambridge CB2-1PZ, UK}

\begin{abstract}
For plane deformations generated by an arbitrary distribution of tractions applied in a small region on the boundary of an elastic half-plane, the rates-of-decay for displacements, stresses and strain energy density are obtained as functions of complexity of the load distribution. The rates-of-decay increase in proportion to the complexity of the load distribution; i.e., they increase with the order of the smallest nonvanishing moment of the traction distribution. In orthotropic materials the elastic moduli differ in two perpendicular directions of principal stiffness; in this case as the modulus ratio $E_{2} / E_{1}$ increases, the angular distributions of the displacement and energy density fields become channeled towards the direction of the larger elastic modulus.
\end{abstract}

Introduction. Self-equilibrated tractions acting on a small region of an elastic solid result in stresses that rapidly decay with increasing distance from the region where the tractions are applied. This behaviour is characteristic of systems with elliptic equations of equilibrium. In practice, general knowledge about the variation of stresses with radial distance from a small region of load application is widely used to claim that at large distances from the loaded region (i.e., large in comparison with the size of the loaded region), the distribution of tractions is unimportant - the only significant effect on the stresses comes from any resultant force and couple that are equivalent to the distributed tractions. If distances are not very large, however, even self-equilibrated tractions generate stresses that may depend on details of the traction distribution. To explain how the stress field depends on the distribution of tractions, we examine the effect of any local distribution of tractions on the rate-of-decay of stresses in an orthotropic elastic solid. The anisotropy of the solid affects the circumferential distribution of stress around the loaded area but not the rate-of-decay. It will be shown that the rate-of-decay (or rate-of-diffusion) of stress depends on the complexity of the traction distribution but is independent of whether the applied tractions act normal or tangential to the loaded surface; i.e., for tractions applied in a small area, the rate-of-decay does not depend on the

Received December 16, 1997.

1991 Mathematics Subject Classification. Primary 35,76.

(C)1999 Brown University 
moment of the applied tractions, but rather on the moment of the spatial distribution of the applied tractions.

The statement that self-equilibrated systems of tractions result in stresses that decay rapidly with distance from the region where tractions are applied is known as St. Venant's Principle; this principle expresses the property of diffusivity in systems represented by elliptic equations of equilibrium and compatibility. Barre St. Venant (1855) proposed this principle on the basis of his analyses of end effects in slender elastic beams and shafts. The principle was generalized to include tractions acting on a body of infinite extent by Boussinesq who stated that, "An equilibrated system of external forces applied to an elastic body, all the points of application lying within a given sphere, produces deformations of negligible magnitude at distances from the sphere which are sufficiently large compared to its radius." While Boussinesq's expression of St. Venant's principle is quite general, it gives no sense of how the rate-of-diffusion depends on details of the traction distribution and/or the configuration of the body.

After examining the stress distribution results from low-order sets of tangential forces acting in a small region on the surface of a half-space, von Mises (1945) noted that the rate-of-decay of stresses did not directly correspond with whether or not the set of forces was self-equilibrated. He proposed that the rate-of-decay depends also on whether the self-equilibrated system of forces is also in astatic equilibrium. (von Mises introduced the term astatic equilibrium to characterise a self-equilibrated set of forces that act at specific points on a surface if this set remains in equilibrium when the forces are rotated through an arbitrary angle $\psi$.) A general proof of Saint-Venant's principle as stated by von Mises was given by Sternberg (1954), who examined an elastic body loaded by surface tractions applied in a small region of radius $\varepsilon$. Sternberg proved that with increasing radius from the region of load application, the decay of stresses is more rapid if the applied forces are not merely self-equilibrated but are also in astatic equilibrium. Proofs of these statements were reviewed by Gurtin 1973 .

Later investigations established an exponential rate-of-decay with axial distance for 2-D stresses generated by self-equilibrated tractions acting over the end of a semi-infinite slender strip or cylinder composed of anisotropic elastic material (Everstine and Pipkin 1971, Horgan 1972, Horgan and Knowles 1983, Horgan and Simmonds 1994, Arimitsu et al 1995, Durban and Stronge 1995, Horgan 1996). For an orthotropic half-plane, Stronge and Kashtalyan 1997 found the distribution of stresses and the rates-of-decay arising from some distributions of traction in a small area on the edge of a half-plane.

1. Stress field from a discrete force. Previous investigations of St. Venant's principle were based on consideration of particular sets of traction distributions involving a small number of discrete forces. Here we consider an orthotropic elastic half-space where 2-D stress and displacement fields result from an arbitrary distribution of tractions acting in a small region $\varepsilon$ on the surface $y=0$.

Let the force $\mathbf{F} \equiv F \mathbf{e}$ act on the surface of an orthotropic elastic half-plane $y<0$ at an angle $\Omega$ from the normal, $\mathbf{e}=\sin \Omega \mathbf{e}_{\mathbf{x}}+\cos \Omega \mathbf{e}_{\mathbf{y}}$ as shown in Fig. 1. The half-plane has Young's moduli $E_{x}$ and $E_{y}$, in directions tangential $\mathbf{e}_{\mathbf{x}}$ and normal $\mathbf{e}_{\mathbf{y}}$ to the surface. For a polar coordinate system with origin at the point of application, the force results in 


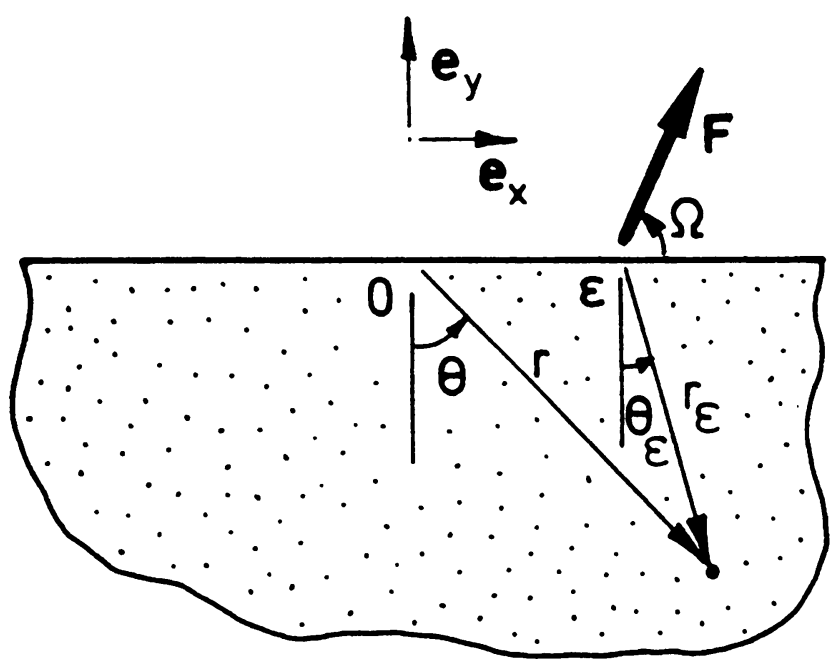

FIG. 1. Force $\mathbf{F}$ on surface of orthotropic half-plane

radial stress only and the stress field can be expressed as

$$
\begin{gathered}
\sigma_{r r}(r, \theta)=\frac{1}{r} \kappa \mathbf{F} \cdot \boldsymbol{\varphi}(\theta), \quad \sigma_{r \theta}=\sigma_{\theta \theta}=0, \\
\kappa=\frac{\left(u_{1}+u_{2}\right)}{\pi}, \\
\varphi(\theta)=\left[\frac{-\sin \theta}{\lambda(\theta)}\right] \mathbf{e}_{\mathbf{x}}+R_{2}^{1 / 2}\left[\frac{\cos \theta}{\lambda(\theta)}\right] \mathbf{e}_{\mathbf{y}}, \\
\lambda(\theta)=\sin ^{4} \theta+2 R_{1} \sin ^{2} \theta \cos ^{2} \theta+R_{2} \cos ^{4} \theta,
\end{gathered}
$$

where $u_{1}$ and $u_{2}$ are roots of a characteristic equation given by Lekhnitskii (1981),

$$
u^{4}-2 R_{1} u^{2}+R_{2}=0
$$

Most orthotropic materials have moduli giving roots $u_{1}, u_{2}$ that are real and distinct but the roots can be complex or, for isotropic materials, real and repeated (Matemilola et. al, 1995). In Eqs. (1) and (2) the parameters $R_{1}$ and $R_{2}$ are ratios of material properties that depend on Young's moduli $E_{x}, E_{y}$, the shear modulus $G$ and Poisson's ratio $\nu_{x y}$. For plane stress these material constants are defined as

$$
R_{1} \equiv \frac{E_{x}}{E_{y}} \frac{E_{y}}{2 G}-\nu_{x y}, \quad R_{2} \equiv \frac{E_{x}}{E_{y}}
$$

In an isotropic material these modulus ratios are identical, $R_{1}=R_{2}=1$. 
If the point of force application is offset a distance $\varepsilon$ along the surface from the origin of the coordinate system, the stress field generated by $\mathbf{F}$ will be

$$
\begin{aligned}
\sigma_{r r} & =\frac{1}{r_{\varepsilon}} \kappa \mathbf{F} \cdot \boldsymbol{\varphi}\left(\theta_{\varepsilon}\right) \cos ^{2}\left(\theta-\theta_{\varepsilon}\right) \\
& =\frac{1}{r} \kappa \mathbf{F} \cdot \boldsymbol{\varphi}_{r r}(\xi, \theta), \\
\sigma_{r \theta} & =\frac{1}{r} \kappa \mathbf{F} \cdot \boldsymbol{\varphi}_{r \theta}(\xi, \theta), \\
\sigma_{\theta \theta} & =\frac{1}{r} \kappa \mathbf{F} \cdot \boldsymbol{\varphi}_{\theta \theta}(\xi, \theta),
\end{aligned}
$$

where $r_{\varepsilon}=\left(r^{2}+\varepsilon^{2}-2 r \varepsilon \sin \theta\right)^{1 / 2}, r_{\varepsilon} \cos \theta_{\varepsilon}=r \cos \theta$, and $\xi \equiv \varepsilon / r$. Here the angular distribution of stress and the offset $\xi$ of the origin from the point of application of the force are represented solely by

$$
\begin{aligned}
\boldsymbol{\varphi}_{r r}(\xi, \theta) & \equiv\left\{\frac{[\xi-\sin \theta][1-\xi \sin \theta]^{2}}{\lambda(\xi, \theta)}\right\} \mathbf{e}_{\mathbf{x}}+R_{2}^{1 / 2}\left\{\frac{[1-\xi \sin \theta]^{2} \cos \theta}{\lambda(\xi, \theta)}\right\} \mathbf{e}_{\mathbf{y}} \\
\boldsymbol{\varphi}_{r \theta}(\xi, \theta) & \equiv\left\{\frac{\xi[-\xi+\sin \theta][1-\xi \sin \theta] \cos \theta}{\lambda(\xi, \theta)}\right\} \mathbf{e}_{\mathbf{x}}+R_{2}^{1 / 2}\left\{\frac{-\xi(1-\xi \sin \theta) \cos ^{2} \theta}{\lambda(\xi, \theta)}\right\} \mathbf{e}_{\mathbf{y}} \\
\boldsymbol{\varphi}_{\theta \theta}(\xi, \theta) & \equiv\left\{\frac{\xi^{2}[\xi-\sin \theta] \cos ^{2} \theta}{\lambda(\xi, \theta)}\right\} \mathbf{e}_{\mathbf{x}}+R_{2}^{1 / 2}\left\{\frac{\xi^{2} \cos ^{3} \theta}{\lambda(\xi, \theta)}\right\} \mathbf{e}_{\mathbf{y}} \\
\lambda(\xi, \theta) & \equiv(-\xi+\sin \theta)^{4}+2 R_{1}(-\xi+\sin \theta)^{2} \cos ^{2} \theta+R_{2} \cos ^{4} \theta
\end{aligned}
$$

It is useful to recognize that both components of the vector function $\boldsymbol{\varphi}_{i j}$ have the same form; i.e., the normal and tangential components can each be expressed as a rational function of the offset $\xi=\varepsilon / r$.

2. Stress field from set of parallel forces. Rather than a single force, consider a set of $n$ parallel forces $\mathbf{F}_{k}=F_{k} \mathbf{e}, k=1, \ldots, n$, each force acting on the surface at a spatial coordinate $\varepsilon_{k}$ relative to the origin of the coordinate system and acting in a direction e. Superposing the effects of these surface tractions gives components of stress

$$
\sigma_{i j}=\frac{1}{r} \kappa \sum_{k=1}^{n} \mathbf{F}_{k} \cdot \boldsymbol{\varphi}_{i j}\left(\xi_{k}, \theta\right) .
$$

If $\xi_{k} \equiv \varepsilon_{k} / r \in(-\xi, \xi)$ and $\xi \ll 1$ the distribution function $\varphi_{i j}$ in the expression above can be expanded in a Maclaurin series in terms of powers of $\xi_{k}$; i.e.,

$$
\boldsymbol{\varphi}_{i j}=\sum_{m=0}^{\infty} \boldsymbol{\Phi}_{i j}^{(m)}(\theta) \xi_{k}^{m}
$$

where the coefficients

$$
\boldsymbol{\Phi}_{i j}^{(m)}(\theta) \equiv \partial^{m} \boldsymbol{\varphi}_{i j} /\left.\partial \xi_{k}^{m}\right|_{\xi_{k}=0}
$$


are independent of the locations $\xi_{k}$ of tractions acting on the loaded area. Substitution into (6) gives

$$
\sigma_{i j}=\frac{1}{r} \kappa \sum_{k=1}^{n} \mathbf{F}_{k} \cdot \sum_{m=0}^{\infty} \frac{\mathbf{\Phi}_{i j}^{(m)}(\theta) \xi_{k}^{m}}{m !} .
$$

After interchanging the order of summation we obtain

$$
\sigma_{i j}=\frac{1}{r} \kappa \sum_{m=0}^{\infty} \frac{\mathbf{e} \cdot \boldsymbol{\Phi}_{i j}^{(m)}(\theta)}{m ! r^{m}} \sum_{k=1}^{n} F_{k} \varepsilon_{k}^{m} .
$$

This expression for stresses arising from a set of $n$ parallel forces acting in the loaded area has a final summation of the first through the $n$th moments of the traction distribution rather than the moment of the tractions.

Hence the smallest rate-of-decay for stresses generated by a system of parallel forces applied in a small region $\varepsilon$ on the boundary of an orthotropic half-plane is determined by the order $M$ of the smallest nonvanishing moment of the traction distribution. For example, if the resultant of the applied forces vanishes, but the first moment of their distribution is not zero, $M=1$ and stresses decrease as $\varepsilon^{M} / r^{M+1}=\varepsilon / r^{2}$, a case represented by line (ii) in Table 1. While von Mises's and Sternberg's distinction between self-equilibrated and astatic self-equilibrated sets of forces is sufficient to describe the rate-of-decay if the order of complexity for the traction distribution is small $(M<3)$, it is an artificial distinction that is not sufficient for more complex traction distributions.

3. Distribution of tractions giving rate-of-decay $\varepsilon^{M} / r^{M+1}$. If a set of $n$ parallel forces acts on the surface of the half-plane and this set gives a smallest rate-of-decay $\varepsilon^{M} / r^{M+1}$, then

$$
0=\sum_{k=1}^{n} F_{k} \xi_{k}^{m}, \quad m=0,1, \ldots, M-1 .
$$

The smallest number of forces that can satisfy this condition is given by $n=M+1$. To determine a distribution of tractions for any specific rate-of-decay $\varepsilon^{M} / r^{M+1}$, let $M+1$ parallel forces of unknown magnitude $\beta_{k} F$ act on the surface at locations $\varepsilon_{k}$ relative to the origin $(k=1, \ldots, M+1)$. The relative magnitude of the forces is given by the weight functions $\beta_{k}$.

First consider a slightly reduced set of $M$ forces. For this system condition (9) gives a system of $M$ simultaneous homogeneous linear equations with respect to the weight functions $\beta_{1}, \ldots, \beta_{M}$ :

$$
\left[\begin{array}{cccc}
1 & 1 & \cdots & 1 \\
\varepsilon_{1} & \varepsilon_{2} & \cdots & \varepsilon_{M} \\
\varepsilon_{1}^{2} & \varepsilon_{2}^{2} & \cdots & \varepsilon_{M}^{2} \\
\vdots & \vdots & \vdots & \vdots \\
\varepsilon_{1}^{M-1} & \varepsilon_{2}^{M-1} & \cdots & \varepsilon_{M}^{M-1}
\end{array}\right]\left\{\begin{array}{c}
\beta_{1} \\
\beta_{2} \\
\beta_{3} \\
\vdots \\
\beta_{M}
\end{array}\right\}=\left\{\begin{array}{c}
0 \\
0 \\
0 \\
\vdots \\
0
\end{array}\right\} .
$$




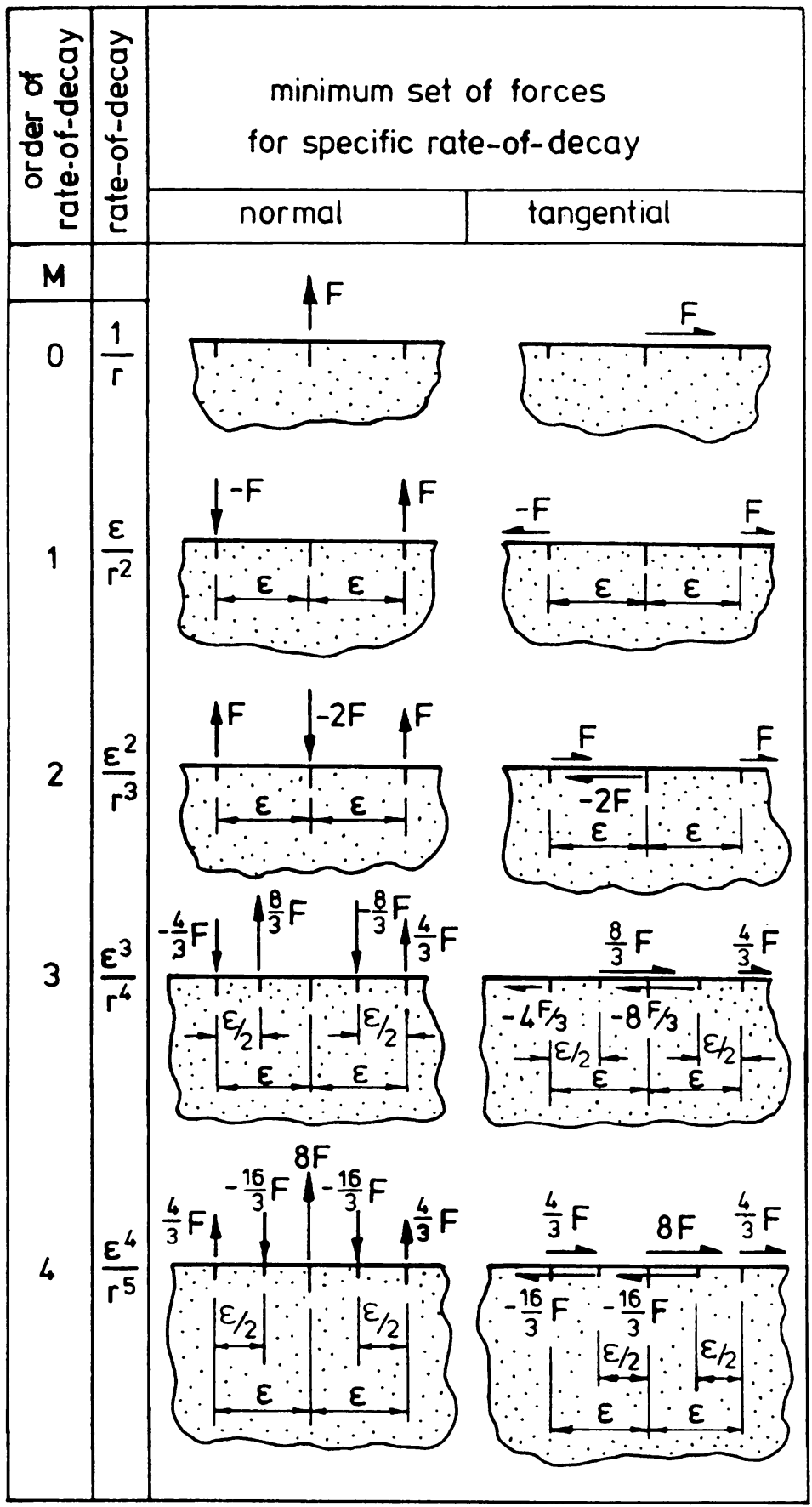

TABle 1 
The coefficients of Eqs. (10) have a determinant

$$
D=\left|\begin{array}{cccc}
1 & 1 & \cdots & 1 \\
\varepsilon_{1} & \varepsilon_{2} & \cdots & \varepsilon_{M} \\
\varepsilon_{1}^{2} & \varepsilon_{2}^{2} & \cdots & \varepsilon_{M}^{2} \\
\vdots & \vdots & \vdots & \vdots \\
\varepsilon_{1}^{M-1} & \varepsilon_{2}^{M-1} & \cdots & \varepsilon_{M}^{M-1}
\end{array}\right|
$$

This is the Vandermonde determinant and it is equal to zero only if two of the distances $\varepsilon_{k}$ are equal. If the distances $\varepsilon_{k}$ are all distinct then the determinant does not vanish $D \neq 0$ and the weight functions $\beta_{k}$ that satisfy (10) are identically zero.

For the minimum number of parallel forces $M+1$, condition (9) gives a system of $M$ simultaneous homogeneous linear equations for $M+1$ weight functions $\beta_{k}$; i.e.,

$$
\left[\begin{array}{cccc}
1 & 1 & \cdots & 1 \\
\varepsilon_{1} & \varepsilon_{2} & \cdots & \varepsilon_{M} \\
\varepsilon_{1}^{2} & \varepsilon_{2}^{2} & \cdots & \varepsilon_{M}^{2} \\
\vdots & \vdots & \vdots & \vdots \\
\varepsilon_{1}^{M-1} & \varepsilon_{2}^{M-1} & \cdots & \varepsilon_{M}^{M-1}
\end{array}\right]\left\{\begin{array}{c}
\beta_{1} \\
\beta_{2} \\
\beta_{3} \\
\vdots \\
\beta_{M}
\end{array}\right\}=-\beta_{M+1}\left\{\begin{array}{c}
1 \\
\varepsilon_{M+1} \\
\varepsilon_{M+1}^{2} \\
\vdots \\
\varepsilon_{M+1}^{M-1}
\end{array}\right\} .
$$

Solving for the weight functions $\beta_{k}$, Eq. (12) gives

$$
\beta_{k}=-\frac{1}{D} \beta_{M+1} \sum_{j=1}^{M}(-1)^{j+k} D_{j k} \varepsilon_{M+1}^{j-1}
$$

where $D$ is the Vandermonde determinant (11) and $D_{j k}$ is the $j k$ minor of this determinant. This expression can be simplified if the final force in the set is designated as the force at the origin, $F_{M+1} \equiv F_{0}$ so that $\varepsilon_{M+1}=0$. Then $\beta_{M+1}=\beta_{0}$ and

$$
\beta_{k}=-\frac{1}{D} \beta_{0}(-1)^{1+k} D_{1 k} .
$$

3.1. An even power $M$. The stresses can be expressed more compactly if the tractions are distributed either symmetrically or antisymmetrically with respect to the origin. Superposition of a system of $n$ parallel traction forces $F_{k}$ applied at points $\varepsilon_{k}$ and a parallel system of $n$ forces $F_{k}^{\prime}$ applied at points $-\varepsilon_{k}$ where $F_{k}^{\prime}\left(-\varepsilon_{k}\right)=F_{k}\left(\varepsilon_{k}\right)$, gives a symmetric system of $2 n$ forces. This even distribution of loading generates a stress field,

$$
\sigma_{i j}=\frac{1}{r} \kappa \sum_{m=0}^{\infty} \frac{\mathbf{e} \cdot \boldsymbol{\Phi}_{i j}^{(2 m)}}{r^{2 m}(2 m) !} \sum_{k=1}^{n} 2 F_{k} \varepsilon_{k}^{2 m}
$$

with odd-powered moments that are identically equal to zero. This symmetric system has a rate-of-decay $\varepsilon^{M} / r^{M+1}$ which is even. For any specified rate-of-decay $M$ that is an even number, the smallest number of forces satisfying condition (9) is $M+2$.

3.2. An odd power $M$. Likewise, superposition of a system of $n$ parallel traction forces $F_{k}$ applied at points $\varepsilon_{k}$ and a parallel system of $n$ forces $F_{k}^{\prime}$ applied at points $-\varepsilon_{k}$ in a manner such that $F_{k}^{\prime}\left(-\varepsilon_{k}\right)=-F_{k}\left(\varepsilon_{k}\right)$, gives an antisymmetric system of $2 n$ forces with 
an odd distribution of loading. This odd distribution of loading generates a stress field,

$$
\sigma_{i j}=\frac{1}{r} \kappa \sum_{m=0}^{\infty} \frac{\mathbf{e} \cdot \boldsymbol{\Phi}_{i j}^{(2 m+1)}}{r^{2 m+1}(2 m+1) !} \sum_{k=1}^{n} 2 F_{k} \varepsilon_{k}^{2 m+1}
$$

with even-powered moments that are identically equal to zero. This antisymmetric system has a rate-of-decay $\varepsilon^{M I} / r^{M+1}$ which is odd. For any specified rate-of-decay $M$ that is an odd number, the smallest number of forces satisfying condition (10) is $M+1$.

4. Effect of anisotropy. In an orthotropic half-space the rate-of-decay of stresses does not depend on material properties. The angular distribution of stresses described by the function $\boldsymbol{\Phi}_{i j}^{(M)}(\theta)$, however, is highly affected by anisotropy. To illustrate this point, distributions of stresses and displacements generated by sets of parallel forces acting on the small region $(-\varepsilon, \varepsilon)$ of the surface will be analysed below for three rates-of-decay $M=1,2,3$.

4.1. Stress field. For different rates-of-decay $M$, the sets of even and odd distributions of parallel forces illustrated in Table 1 result in stresses

$$
\begin{array}{ll}
M=1, & \sigma_{i j}=2 \frac{\varepsilon}{r^{2}} \kappa F \mathbf{e} \cdot \boldsymbol{\Phi}_{i j}^{(1)}(\theta), \\
M=2, & \sigma_{i j}=2 \frac{\varepsilon^{2}}{2 ! r^{3}} \kappa F \mathbf{e} \cdot \boldsymbol{\Phi}_{i j}^{(2)}(\theta), \\
M=3, & \sigma_{i j}=2 \frac{\varepsilon^{3}}{3 ! r^{4}} \kappa F \mathbf{e} \cdot \boldsymbol{\Phi}_{i j}^{(3)}(\theta) .
\end{array}
$$

The angular distributions $\boldsymbol{\Phi}_{i j}^{(M)}(\theta)$ have components that can be expressed in terms of $\lambda(\theta)$ and derivatives of this function. The tangential component is obtained as

$$
\begin{aligned}
\mathbf{e}_{x} \cdot \boldsymbol{\Phi}_{r r}^{(1)}= & \frac{1}{\lambda^{2}}\left\{\lambda\left(1+2 \sin ^{2} \theta\right)-\sin \theta \frac{\partial \lambda}{\partial \sin \theta}\right\} \\
\mathbf{e}_{x} \cdot \boldsymbol{\Phi}_{r \theta}^{(1)}= & \frac{\cos \theta \sin \theta}{\lambda} \\
\mathbf{e}_{x} \cdot \boldsymbol{\Phi}_{\theta \theta}^{(1)}= & 0 \\
\mathbf{e}_{x} \cdot \boldsymbol{\Phi}_{r r}^{(2)}= & \frac{-2}{\lambda^{3}}\left\{\lambda^{2}\left(2 \sin \theta+\sin ^{3} \theta\right)-\lambda\left(1+2 \sin ^{2} \theta\right) \frac{\partial \lambda}{\partial \sin \theta}-\frac{\lambda \sin \theta}{2} \frac{\partial^{2} \lambda}{\partial(\sin \theta)^{2}}\right\} \\
\mathbf{e}_{x} \cdot \boldsymbol{\Phi}_{r \theta}^{(2)}= & \frac{-2 \cos \theta}{\lambda^{2}}\left\{\lambda\left(1+\sin ^{2} \theta\right)-\sin \theta \frac{\partial \lambda}{\partial \sin \theta}\right\} \\
\mathbf{e}_{x} \cdot \boldsymbol{\Phi}_{\theta \theta}^{(2)}= & \frac{-2 \cos { }^{2} \theta \sin \theta}{\lambda}, \\
\mathbf{e}_{x} \cdot \boldsymbol{\Phi}_{r r}^{(3)}= & \frac{6}{\lambda^{4}}\left\{\lambda^{3} \sin ^{2} \theta-\lambda^{2}(2 \sin \theta+\sin 3 \theta) \frac{\partial \lambda}{\partial \sin \theta}\right. \\
& -\frac{\lambda^{2}}{2}\left(1+2 \sin { }^{2} \theta\right) \frac{\partial^{2} \lambda}{\partial(\sin \theta)^{2}}-\frac{\lambda^{2}}{6} \sin \theta \frac{\partial^{3} \lambda}{\partial(\sin \theta)^{3}}+\lambda\left(1+\sin ^{2} \theta\right)\left(\frac{\partial \lambda}{\partial \sin \theta}\right)^{2} \\
& \left.-\sin \theta\left(\frac{\partial \lambda}{\partial \sin \theta}\right)^{3}+\lambda \sin \theta \frac{\partial \lambda}{\partial \sin \theta} \frac{\partial^{2} \lambda}{\partial(\sin \theta)^{2}}\right\}
\end{aligned}
$$




$$
\begin{aligned}
& \mathbf{e}_{x} \cdot \boldsymbol{\Phi}_{r \theta}^{(3)}=\frac{6 \cos \theta}{\lambda^{3}}\left\{\frac{\lambda^{2}}{2} \sin \theta \frac{\partial^{2} \lambda}{(\partial \sin \theta)^{2}}+\left(1+\sin ^{2} \theta\right) \frac{\partial \lambda}{\partial \sin \theta}+\lambda \sin \theta\right\}, \\
& \mathbf{e}_{x} \cdot \boldsymbol{\Phi}_{\theta \theta}^{(3)}=\frac{6 \cos ^{2} \theta}{\lambda^{2}}\left\{\lambda-\sin \theta \frac{\partial \lambda}{\partial \sin \theta}\right\},
\end{aligned}
$$

while the normal components of the angular distribution function for the three lowestorder rates-of-decay are

$$
\begin{aligned}
& \mathbf{e}_{y} \cdot \boldsymbol{\Phi}_{r r}^{(1)}=\frac{-R_{2}^{1 / 2} \cos \theta}{\lambda^{2}}\left\{2 \lambda \sin \theta-\frac{\partial \lambda}{\partial \sin \theta}\right\}, \\
& \mathbf{e}_{y} \cdot \boldsymbol{\Phi}_{r \theta}^{(1)}=\frac{-R_{2}^{1 / 2} \cos ^{2} \theta}{\lambda}, \\
& \mathbf{e}_{y} \cdot \boldsymbol{\Phi}_{\theta \theta}^{(1)}=0 \text {, } \\
& \mathbf{e}_{y} \cdot \boldsymbol{\Phi}_{r r}^{(2)}=\frac{2 R_{2}^{1 / 2} \cos \theta}{\lambda^{3}}\left\{\lambda^{2} \sin ^{2} \theta-2 \lambda \sin \theta \frac{\partial \lambda}{\partial \sin \theta}-\frac{\lambda}{2} \frac{\partial^{2} \lambda}{\partial(\sin \theta)^{2}}+\left(\frac{\partial \lambda}{\partial \sin \theta}\right)^{2}\right\}, \\
& \mathbf{e}_{y} \cdot \boldsymbol{\Phi}_{r \theta}^{(2)}=\frac{2 R_{2}^{1 / 2} \cos ^{2} \theta}{\lambda^{2}}\left\{\lambda \sin \theta-\frac{\partial \lambda}{\partial \sin \theta}\right\} \\
& \mathbf{e}_{y} \cdot \boldsymbol{\Phi}_{\theta \theta}^{(2)}=\frac{2 R_{2}^{1 / 2} \cos ^{3} \theta}{\lambda}, \\
& \mathbf{e}_{y} \cdot \boldsymbol{\Phi}_{r r}^{(3)}=-\frac{6 R_{2}^{1 / 2} \cos \theta}{\lambda^{4}}\left\{-\lambda^{2} \sin ^{2} \theta \frac{\partial \lambda}{\partial \sin \theta}-\lambda^{2} \sin \theta \frac{\partial^{2} \lambda}{\partial(\sin \theta)^{2}}-\frac{\lambda^{2}}{6} \frac{\partial^{3} \lambda}{\partial(\sin \theta)^{3}}\right. \\
& \left.+\lambda \frac{\partial \lambda}{\partial \sin \theta} \frac{\partial^{2} \lambda}{\partial(\sin \theta)^{2}}+2 \lambda \sin \theta\left(\frac{\partial \lambda}{\partial \sin \theta}\right)^{2}-\left(\frac{\partial \lambda}{\partial \sin \theta}\right)^{3}\right\}, \\
& \mathbf{e}_{y} \cdot \boldsymbol{\Phi}_{r \theta}^{(3)}=-\frac{6 R_{2}^{1 / 2} \cos ^{2} \theta}{\lambda^{3}}\left\{-\lambda \sin \theta \frac{\partial \lambda}{\partial \sin \theta}-\frac{\lambda}{2} \frac{\partial^{2} \lambda}{\partial(\sin \theta)^{2}}+\left(\frac{\partial \lambda}{\partial \sin \theta}\right)^{2}\right\}, \\
& \mathbf{e}_{y} \cdot \boldsymbol{\Phi}_{\theta \theta}^{(3)}=-\frac{6 R_{2}^{1 / 2} \cos ^{3} \theta}{\lambda^{2}}\left(\frac{-\partial \lambda}{\partial \sin \theta}\right) \text {. }
\end{aligned}
$$

These stresses and the elastic moduli are used to obtain the distribution of strain energy density $w^{(M)}$ for the $M$ th rate-of-decay of stresses. This strain energy density takes the form

$$
w^{(M)}=2 \kappa^{2} F^{2} \frac{\varepsilon^{2 m}}{r^{2(M+1)}} \mathbf{e} \cdot \mathbf{f}^{(M)}(\theta) .
$$

Figures 2-4 show polar plots of contours of constant strain energy density for the three smallest rates-of-decay and three different sets of material properties: (a) isotropic properties where necessarily $R_{1}=R_{2}=1$; (b) orthotropic properties with the larger elastic modulus in the direction tangential to the surface; and (c) orthotropic properties with the larger elastic modulus perpendicular to the surface. Moduli of the orthotropic materials have been chosen to be representative of fibre-reinforced composites with fibres parallel and perpendicular to the surface of the half-space, respectively. The orthotropic materials have contours of constant strain energy density that are elongated in the direction of fibre reinforcement; i.e., the direction of largest elastic modulus. This effect has 

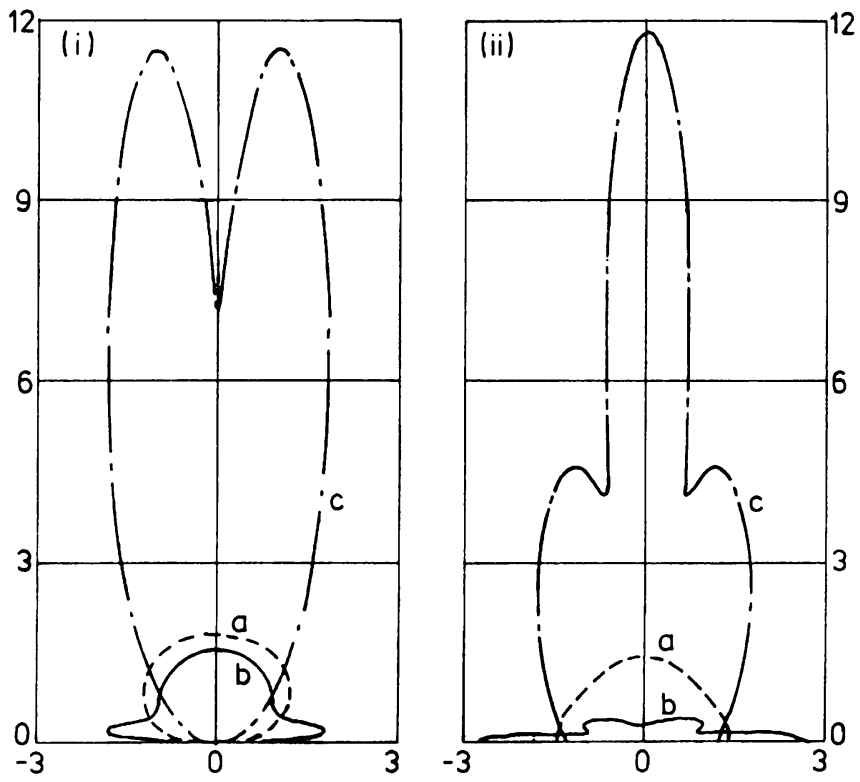

FIG. 2. Contours of constant strain energy density for sets of (i) normal and (ii) tangential forces with rate-of-decay $M=1$. Material parameters: (a) isotropic, $R_{1}=R_{2}=1$; (b) orthotropic material, $R_{1}=R_{2}=20$ (e.g., $E_{x} / E_{y}=20, E_{y} / G=2.03, \nu_{x y}=0.3$ ); (c) orthotropic material, $R_{1}=R_{2}=0.05$ (e.g., $E_{x} / E_{y}=0.05, E_{y} / G=$ $\left.52, \nu_{x y}=0.3\right)$.

been termed stress channeling by Everstine and Pipkin (1971) and Arimitsu et al (1995). The distortion of the stress field by orthotropic elastic moduli is more pronounced for a large modulus perpendicular to the surface. The shape of the contours becomes more uneven as the complexity of the load distribution and rate-of-decay increase.

4.2. Displacement field. The displacement field associated with each rate-of-decay can be obtained by integration of strains. For example, the radial strain $\varepsilon_{r r}$ can be expressed as

$$
\varepsilon_{r r} \equiv \frac{\partial u_{r}}{\partial r}=a_{11} \sigma_{r r}+a_{12} \sigma_{\theta \theta}+a_{16} \sigma_{r \theta}
$$

where the elastic moduli $a_{11}, a_{12}, a_{16}$ are related to the moduli of the orthotropic material and the orientation angle $\theta$. For plane stress,

$$
\begin{aligned}
& a_{11}=\frac{1}{E_{x}}\left(\sin ^{4} \theta+2 R_{1} \sin ^{2} \theta \cos ^{2} \theta+R_{2} \cos ^{4} \theta\right) \\
& a_{12}=\frac{1}{E_{x}}\left(1-2 R_{1}+R_{2}\right) \sin ^{2} \theta \cos ^{2} \theta-\frac{\nu_{x y}}{E_{x}} \\
& a_{16}=\frac{1}{E_{x}}\left[2\left(R_{2} \cos ^{2} \theta-\sin ^{2} \theta\right)-2 R_{1}\left(\cos ^{2} \theta-\sin ^{2} \theta\right) \sin \theta \cos \theta\right] .
\end{aligned}
$$



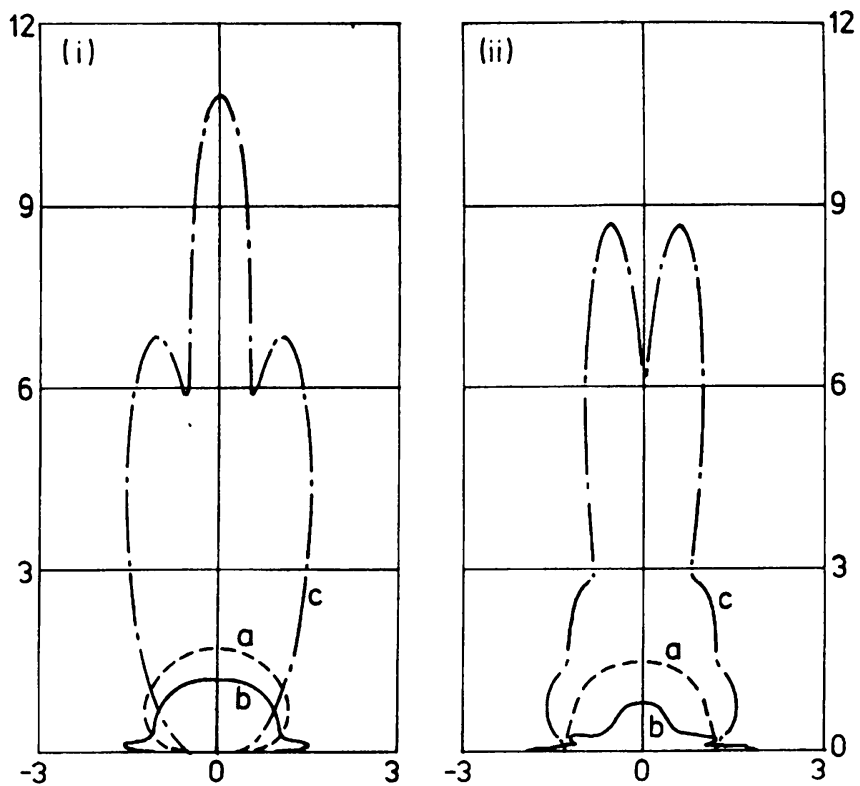

FIG. 3. Contours of constant strain energy density for sets of (i) normal and (ii) tangential forces with rate-of-decay $M=2$. Material parameters: (a) isotropic, $R_{1}=R_{2}=1$; (b) orthotropic material, $R_{1}=R_{2}=20$ (e.g., $E_{x} / E_{y}=20, E_{y} / G=2.03, \nu_{x y}=0.3$ ); (c) orthotropic material, $R_{1}=R_{2}=0.05$ (e.g., $E_{x} / E_{y}=0.05, E_{y} / G=$ $\left.52, \nu_{x y}=0.3\right)$.

After noting that $a_{11}=E_{r}^{-1}=\lambda(\theta) / E_{x}$, an integration gives the following radial displacement $u_{r}(\theta)$ for each set of surface forces:

$$
\begin{array}{ll}
M=1, & u_{r}=-\frac{2 F}{E_{x}}\left(\frac{\varepsilon}{r}\right) \kappa \mathbf{e} \cdot \boldsymbol{\Phi}_{r r}^{\prime(1)}(\theta), \\
M=2, & u_{r}=-\frac{2 F}{2 ! E_{x}}\left(\frac{\varepsilon^{2}}{2 r^{2}}\right) \kappa \mathbf{e} \cdot\left(\boldsymbol{\Phi}_{r r}^{\prime(2)}-\nu_{x y} \boldsymbol{\Phi}_{\theta \theta}^{(2)}\right), \\
M=3, & u_{r}=-\frac{2 F}{3 ! E_{x}}\left(\frac{\varepsilon^{3}}{3 r^{3}}\right) \kappa \mathbf{e} \cdot\left(\boldsymbol{\Phi}_{r r}^{\prime(3)}-\nu_{x y} \mathbf{\Phi}_{\theta \theta}^{(3)}\right),
\end{array}
$$

where the radial component of the angular variation $\boldsymbol{\Phi}_{r r}^{\prime(M)}(\theta), M=1,2,3$, has a tangential component,

$$
\begin{aligned}
\mathbf{e}_{x} \cdot \boldsymbol{\Phi}_{r r}^{\prime(1)}= & \frac{1}{\lambda}\left\{\lambda\left(1+4 \sin ^{2} \theta\right)-\frac{3}{2} \sin \theta \frac{\partial \lambda}{\partial \sin \theta}\right\} \\
\mathbf{e}_{x} \cdot \boldsymbol{\Phi}_{r r}^{(2)}= & \frac{2}{\lambda^{2}}\left\{-\lambda^{2}\left(5 \sin \theta+3 \sin ^{3} \theta\right)-\lambda \sin ^{3} \theta+\lambda\left(\frac{3}{2}-5 \sin ^{2} \theta\right) \frac{\partial \lambda}{\partial \sin \theta}\right. \\
& \left.+\frac{\lambda}{2} \sin \theta \frac{\partial^{2} \lambda}{\partial(\sin \theta)^{2}}-\frac{3}{2} \sin \theta\left(\frac{\partial \lambda}{\partial \sin \theta}\right)^{2}\right\}
\end{aligned}
$$



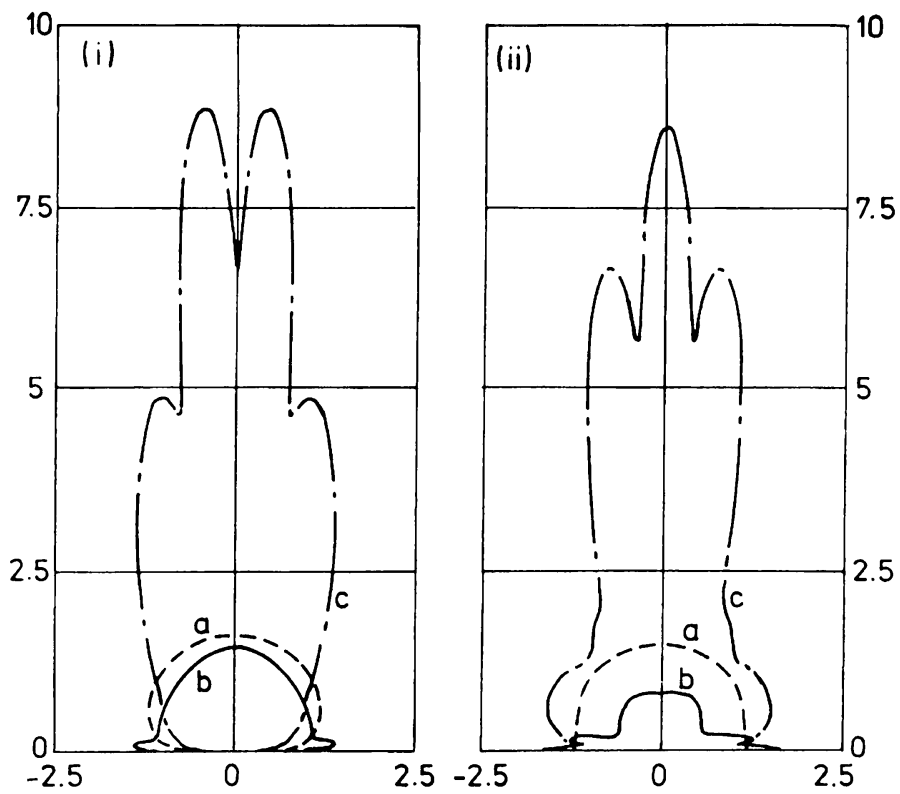

FIG. 4. Contours of constant strain energy density for sets of (i) normal and (ii) tangential forces with rate-of-decay $M=3$. Material parameters: (a) isotropic, $R_{1}=R_{2}=1$; (b) orthotropic material, $R_{1}=R_{2}=20$ (c.g., $E_{x} / E_{y}=20, E_{y} / G=2.03, \nu_{x y}=0.3$ ); (c) orthotropic material, $R_{1}=1.0, R_{2}=0.05$ (e.g., $E_{x} / E_{y}=0.05$, $\left.E_{y} / G=52, \nu_{x y}=0.3\right)$.

$$
\begin{aligned}
\mathbf{e}_{x} \cdot \boldsymbol{\Phi}_{r r}^{\prime(3)}= & \frac{6}{\lambda^{3}}\left\{2 \lambda^{3} \sin ^{2} \theta-2 \lambda^{2}\left(\sin \theta+\sin ^{3} \theta\right) \frac{\partial \lambda}{\partial \sin \theta}\right. \\
& -\frac{\lambda^{2}}{2}\left(1+2 \sin ^{2} \theta\right) \frac{\partial^{2} \lambda}{\partial(\sin \theta)^{2}}-\frac{\lambda^{2}}{6} \sin \theta \frac{\partial^{3} \lambda}{\partial(\sin \theta)^{3}} \\
& +\lambda\left(\frac{3}{2}+\sin ^{2} \theta\right)\left(\frac{\partial \lambda}{\partial \sin \theta}\right)^{2}-\sin \theta\left(\frac{\partial \lambda}{\partial \sin \theta}\right)^{3} \\
& +\frac{3 \lambda}{4} \sin \theta \frac{\partial \lambda}{\partial \sin \theta} \frac{\partial^{2} \lambda}{\partial(\sin \theta)^{2}}+3 \lambda^{2} \sin ^{2} \theta+\lambda^{3} \sin ^{3} \theta \frac{\partial^{2} \lambda}{\partial(\sin \theta)^{2}} \\
& \left.+\lambda\left(\frac{3}{2} \sin \theta+\sin ^{3} \theta\right) \frac{\partial \lambda}{\partial \sin \theta}-\frac{1}{2}\left(1+\sin ^{2} \theta\right)\left(\frac{\partial \lambda}{\partial \sin \theta}\right)^{2}\right\}
\end{aligned}
$$

and normal components

$$
\begin{aligned}
& \mathbf{e}_{y} \cdot \boldsymbol{\Phi}_{r r}^{(1)}=\frac{R_{2}^{1 / 2} \cos \theta}{\lambda}\left\{-4 \lambda \sin \theta+\frac{3}{2} \frac{\partial \lambda}{\partial \sin \theta}\right\}, \\
& \mathbf{e}_{y} \cdot \boldsymbol{\Phi}_{r r}^{\prime(2)}=\frac{2 R_{2}^{1 / 2} \cos \theta}{\lambda^{2}}\left\{5 \lambda^{2} \sin ^{2} \theta+\lambda \sin ^{2} \theta-5 \lambda \sin \theta \frac{\partial \lambda}{\partial \sin \theta}\right.
\end{aligned}
$$



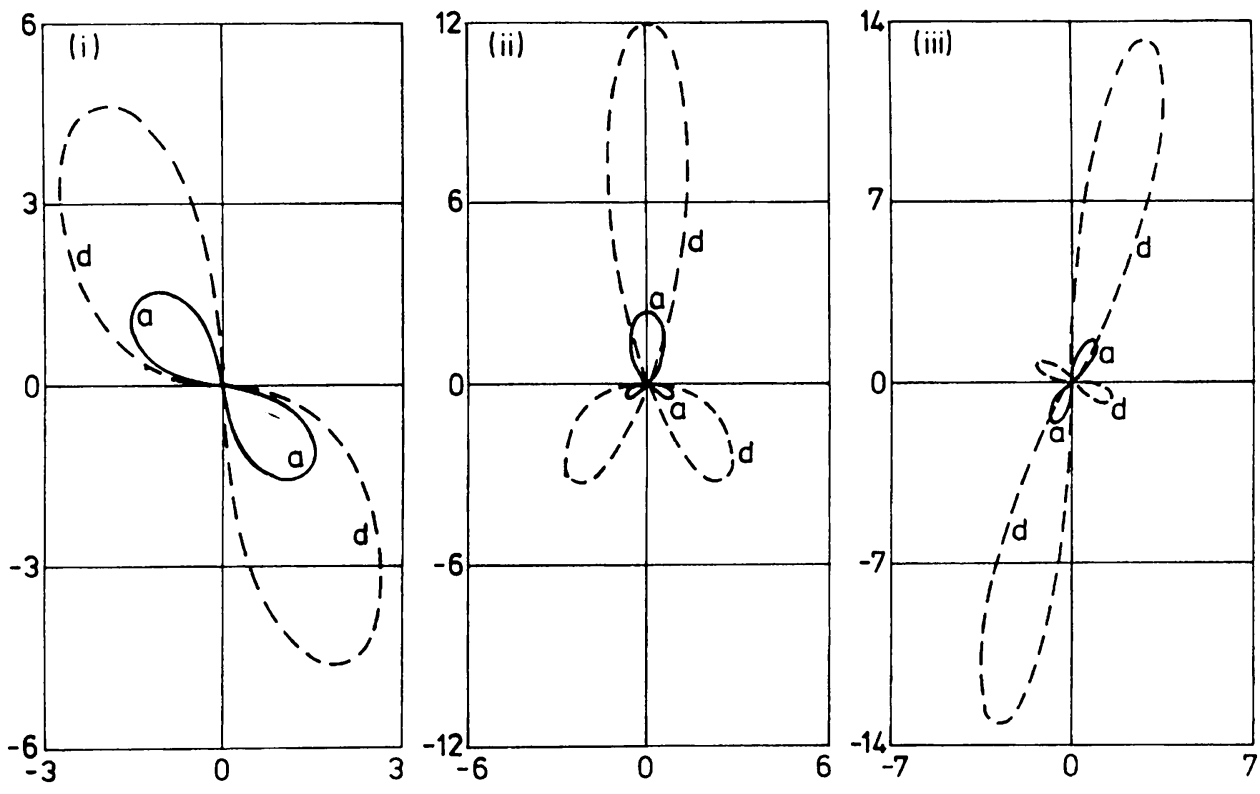

FIG. 5. Contours of constant radial displacement $u_{r}(\theta)$ for sets of normal forces with rates-of-decay (i) $M=1$; (ii) $M=2$; and (iii) $M=3$. The material parameters are (a) isotropic, $R_{1}=R_{2}=1$; (d) orthotropic material, $R_{1}=1.0, R_{2}=0.5$ (e.g., $E_{x} / E_{y}=0.5$, $\left.E_{y} / G=5.2, \nu_{x y}=0.3\right)$.

$$
\begin{aligned}
& \left.-\frac{1}{2} \lambda \frac{\partial^{2} \lambda}{\partial(\sin \theta)^{2}}+\frac{3}{2}\left(\frac{\partial \lambda}{\partial \sin \theta}\right)^{2}\right\} \\
\mathbf{e}_{y} \cdot \boldsymbol{\Phi}_{r r}^{\prime(3)}= & \frac{6 R_{2}^{1 / 2} \cos \theta}{\lambda^{2}}\left\{\frac{\lambda^{2}}{6} \frac{\partial^{3} \lambda}{\partial(\sin \theta)^{3}}+2 \lambda^{2} \sin \theta \frac{\partial^{2} \lambda}{\partial(\sin \theta)^{2}}-\frac{5 \lambda}{4} \frac{\partial \lambda}{\partial \sin \theta} \frac{\partial^{2} \lambda}{\partial(\sin \theta)^{2}}\right. \\
& \left.+\frac{3}{2}\left(\frac{\partial \lambda}{\partial \sin \theta}\right)^{3}-4 \lambda \sin \theta\left(\frac{\partial \lambda}{\partial \sin \theta}\right)^{2}-\lambda(1-2 \lambda) \sin ^{2} \theta \frac{\partial \lambda}{\partial \sin \theta}\right\} .
\end{aligned}
$$

In order to illustrate the effect of anisotropy on the distribution of the radial displacement, Fig. 5 shows polar plots of a contour of constant displacement for different sets of normal force and two different materials. The orthotropic material simulates a fibre reinforced material with the largest modulus perpendicular to the surface. To make the plots more readable, positive displacements are shown in the upper half-plane and negative displacements in the lower half-plane. In each case the displacement field has a number of lobes equal to the rate-of-decay plus one. For the orthotropic material the lobes show elongation in the direction of largest modulus. The difference between the amplitude of lobes for distinct materials increases with increasing rate-of-decay; i.e., the effect of orthotropy on ellipticity of the displacement field increases as the order of the rate-ofdecay increases. 
Conclusion. For a load $\mathbf{F}$ that is distributed over length $2 \varepsilon$ on the surface of a halfspace, the stress field $\sigma_{i j}$ has terms with magnitude proportional to $|\mathbf{F}| / \varepsilon$ that decay as $1 / r$, terms with magnitude proportional to the first moment of the force distribution that decay as $\varepsilon / r^{2}$, terms with magnitude proportional to the second moment of the force distribution that decay as $\varepsilon^{2} / r^{3}$, etc. This result applies also if the tractions are applied at the tip of an elastic wedge as long as the half-angle of the wedge is no greater than $\pi / 2$ (Markenscoff, 1994) and it applies for tractions applied in an interior region that is far from any boundaries. In these cases of planar deformations, tractions that act in a region of radius $\varepsilon$ in an elastic solid result in stresses $\sigma_{i j}$ that decrease as $\varepsilon^{M} / r^{M+1}$ where $M$ is the order of the smallest nonvanishing moment of the traction distribution for any component of the applied tractions. As a straightforward extension we make a conjecture that for a three-dimensional stress field resulting from tractions acting in a spherical region of radius $\varepsilon$, the stresses decrease as $\varepsilon^{M} / r^{M+2}$ where $M$ is the order of the smallest nonvanishing component of the moment of the traction distribution for any component of the applied tractions. These stress variations apply at distances that are large in comparison with the size of the loaded area $r / \varepsilon \gg 1$. Thus it is not whether or not the tractions are self-equilibrated that determines the rate-of-diffusion. but rather the smallest nonvanishing moment of any component of the applied traction distribution. This distinction is especially important in considering tangential rather than normal tractions.

The angular distribution of stresses in the half-plane for any particular rate-of-decay depends on the direction of loading given by the unit vector e as well as the anisotropy of the half-plane. In other words, if two systems of parallel forces act on the same solid and they have both the same rate-of-decay and the same direction, then they have the same angular distribution of stresses. Increasing anisotropy results in increased stress channeling; i.e., the stress distribution becomes more extensive in the direction of largest Young's modulus.

\section{REFERENCES}

[1] Y. Arimitsu, K. Nishioka, and T. Senda, A study of Saint-Venant's principle for composite materials by means of internal stress fields, ASME J. Appl. Mechanics 62, 53- 58 (1995)

[2] D. Durban and W. J. Stronge, Plane strain incremental response and sensitivity of stretched plates, European J. Mechanics - Solids 14, 553-575 (1995)

[3] G. C. Everstine and A. C. Pipkin, Stress channeling in transversely isotropic composites, J. Elasticity 2, 335-339 (1971)

[4] M. E. Gurtin, The linear theory of elasticity, Handbuch der Physik (ed. S. Flugge), 1973, pp. 190207

[5] C. O. Horgan, Some remarks on Saint-Venant's principle for transversely isotropic composites, J. of Elasticity 2, 335339 (1972)

[6] C. O. Horgan, Recent developments concerning Saint-Venant's principle: A second update, Appl. Mech. Reviews 49(10), S101-111 (1996)

[7] C. O. Horgan and J. K. Knowles, Recent developments concerning Saint-Venant's principle, Advances in Applied Mechanics, Vol. 23, Academic Press, 1983, pp. 179-267

[8] C. O. Horgan and J. G. Simmonds, Saint Venant end effects in composite structures, Composites Engineering 3, 279286 (1994)

[9] S. G. Lekhnitskii, Theory of Elasticity of an Anisotropic Elastic Body, translated from the Russian edition, Holden-Day, Inc., San Francisco, 1981 
[10] X. Markenscoff, Some remarks on the wedge paradox and Saint Venant's principle, ASME J. Appl. Mechanics 61, 519-523 (1994)

[11] S. A. Matemilola, W. J. Stronge, and D. Durban, Diffusion rate for stress in orthotropic materials, ASME J. Appl. Mechanics 62, 654-661 (1995)

[12] R. von Mises, On Saint-Venant's principle, Bull. American Mathematical Society 51, 555-562 (1945)

[13] E. Sternberg, On Saint-Venant's principle, Quart. Appl. Math. 11, 393-402 (1954)

[14] W. J. Stronge and M. Kashtalyan, St. Venant's principle for two-dimensional anisotropic elasticity, Acta Mechanica 124, 213-218 (1997)

[15] R. A. Toupin, Saint-Venant's Principle, Arch. Rat. Mech. Analysis 18, 83-96 (1965) 